\title{
Case Report: Angiostrongylus cantonesis Myelitis in Thailand
}

\author{
Tharatorn Nuntawit, Wantin Sribenjalux, ${ }^{\star}$ and Atibordee Meesing \\ Division of Infectious Diseases and Tropical Medicine, Department of Medicine, Faculty of Medicine, Khon Kaen University, Khon Kaen, Thailand
}

\begin{abstract}
A 67-year-old man presented with headache, middle back pain that radiated to both legs, and paresthesia in the right leg for 1 day. He had eaten raw shrimp 1 week previously. Over the next week after admission, he developed urinary retention and weakness in both legs. The numbness in his right leg expanded to below the umbilicus. Magnetic resonance imaging of the spinal cord showed myelopathy with minimal cord swelling at T9 to the conus medullaris and a hemorrhagic lesion from T10 to T11. A complete blood count on day 28 after the onset of symptoms revealed leukocytosis without eosinophilia and no white blood cells in his cerebrospinal fluid. Results of an immunochromatographic test kit were positive for Angiostrongylus cantonesis but negative for Gnathostoma spinigerum. After a 4-week course of albendazole combined with a tapering dose of dexamethasone, he achieved nearly complete recovery.
\end{abstract}

\section{INTRODUCTION}

Angiostrongylus cantonensis, also called rat lungworm, is a parasitic nematode and a leading cause of eosinophilic meningitis. Southeast Asia and tropical Pacific islands are considered endemic areas for this parasite, but there have been an increasing number of cases reported in South and Central America, the Caribbean, the United States, and Australia. ${ }^{1,2}$ In Thailand, approximately $100 \mathrm{~A}$. cantonensis infections are reported annually, and the prevalence is particularly high in the northeast region. ${ }^{3} A$. cantonensis completes its lifecycle in snails and Rattus rats, but many animals, such as freshwater shrimp, coconut crabs, frogs, and slugs, can be intermediate hosts. Ingestion of raw or undercooked animals or fresh vegetables contaminated with larvae from snails or slugs can cause infection in humans. ${ }^{4}$ Patients infected with $A$. cantonensis typically present with meningitis, which is accompanied by symptoms such as headache, photophobia, stiff neck, abnormal skin sensation, nausea, vomiting, fatigue, and body aches. ${ }^{5}$ A cantonensis myelitis is rare and the diagnosis is often delayed because of under-recognition. We report a case of $A$. cantonensis myelitis encountered during October 2020 at Srinagarind Hospital in Khon Kaen, Thailand.

\section{CASE PRESENTATION}

A 67-year-old man with hypertension and hyperuricemia presented at a local hospital after experiencing headache, middle back pain that radiated to both legs, and paresthesia in the right leg for 1 day. He had eaten raw shrimp 1 week previously. After admission, he developed urinary retention and weakness of both legs that started in the right leg and then progressing to the left within 1 week. The area of numbness also expanded to the right side of the trunk below the umbilicus. He was bedridden and required intravenous morphine to alleviate the severe back pain. The pain resolved spontaneously within a few days, but the other neurological symptoms continued to worsen. Magnetic resonance imaging (MRI) of the thoracic and lumbar spine was performed during week 3 of admission and revealed myelopathy at T9 to the conus medullaris with spinal cord hemorrhage at T11-T12 and minimal spinal cord

*Address correspondence to Wantin Sribenjalux, Division of Infectious Diseases and Tropical Medicine, Department of Medicine, Faculty of Medicine, Khon Kaen University, Khon Kaen, 40002, Thailand. E-mail: wantsr@kku.ac.th swelling (Figures 1 and 2). The patient was subsequently referred for further investigation.

On the day of hospitalization at Srinagarind Hospital, his vital signs were as follows: blood pressure, 126/84 mmHg; heart rate, $108 / \mathrm{min}$; body temperature, $38.0^{\circ} \mathrm{C}$; and respiratory rate, $20 / \mathrm{min}$. Neurological examination revealed muscle strength with grade $3 / 5$ in the right lower limb, grade $4 / 5$ in the left lower limb, and grade 5/5 in the upper limbs. Impaired pinprick sensations at the right side of trunk below the umbilical area and right lower limb were reported. Loose sphincter tone and diminished knee and ankle jerks were also detected bilaterally. Other physical examination findings were unremarkable.

Cerebrospinal fluid (CSF) examination on day 28 after the onset of symptoms showed a red blood cell count of 390 cell $/ \mathrm{mm}^{3}$ and white blood cell count of 0 cell $/ \mathrm{mm}^{3}$. His sugar and protein values were $82 \mathrm{mg} / \mathrm{dL}$ and $60 \mathrm{mg} / \mathrm{dL}$, respectively. CSF Gram staining, bacterial culture, india ink staining, cryptococcal antigen, acid-fast bacilli staining, and polymerase chain reaction (PCR) assay for mycobacteria yielded negative results. A complete blood count $(\mathrm{CBC})$ revealed leukocytosis without eosinophilia. Other blood chemistry findings were unremarkable. The results of the serum assay for Angiostrongylus cantonesis antibody that was performed using an immunochromatographic technique were positive, but the results of the test for Gnathostoma spinigerum were negative. The CSF was subjected to $A$. cantonesis-specific and G. spinigerumspecific PCR assays, ${ }^{6,7}$ but the results of both tests were negative.

The patient was treated with albendazole $800 \mathrm{mg} /$ day for 4 weeks plus oral dexamethasone starting at $12 \mathrm{mg} /$ day; then, it was tapered over the course of 4 weeks. At the 4 -week followup examination, the patient's urinary retention had completely resolved and his muscle strength had significantly improved. Additionally, he had gone from being bedridden to being able to walk slowly with a walking aid.

\section{DISCUSSION}

We encountered a case of myelitis as an atypical presentation of $A$. cantonesis. Most cases of angiostrongyliasis present with eosinophilic meningitis lasting from 7 to 14 days. Patients usually have moderate to severe headache and are afebrile. Neck stiffness is detected in only $30 \%$ to $50 \%$ of patients; therefore, $A$. cantonesis infection cannot be excluded only if there is no sign of meningeal irritation. Although other forms 


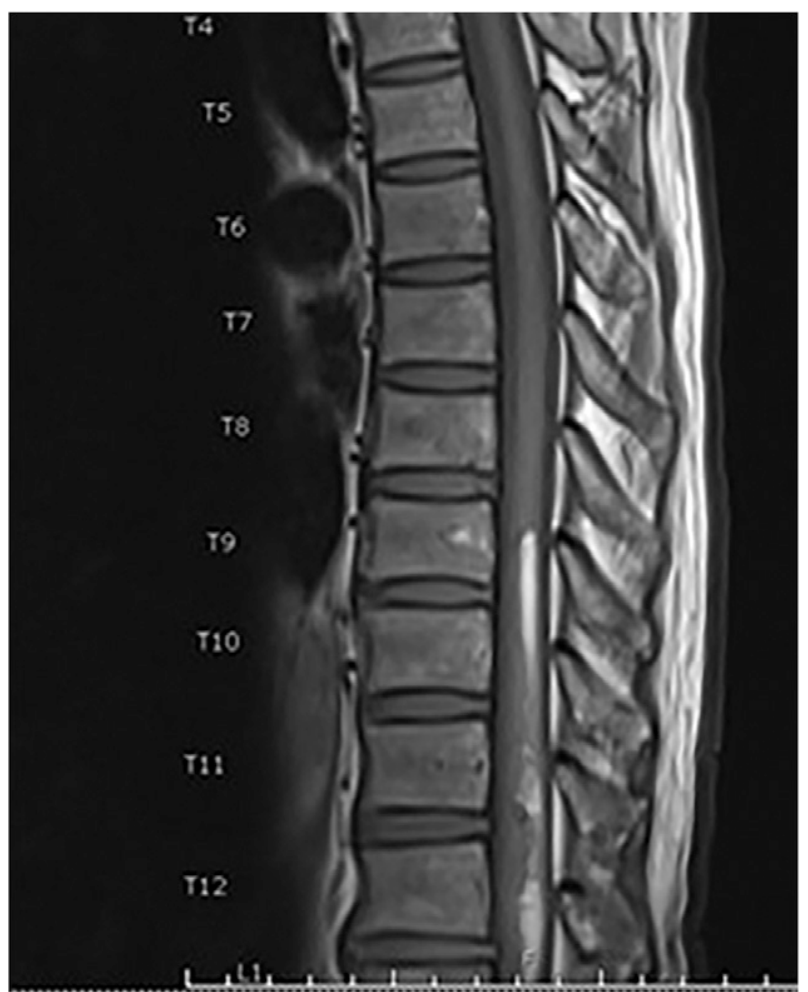

Figure 1. Hyperintensity lesion at T9-T12 in the T1 sagittal view of the T-spine using magnetic resonance imaging (MRI).

of angiostrongyliasis are rare, ocular angiostrongyliasis has been occasionally reported. ${ }^{8} A$ thorough evaluation of the patient's history is an important part of the diagnosis because most infected patients have ingested freshwater raw snails or other intermediate hosts within 7 to 30 days before the onset of symptoms. ${ }^{5}$ Several other case reports of patients with $A$. cantonesis myelitis in the existing literature are listed along with their clinical presentations, diagnostic methods, treatment, and prognoses in Table 1.

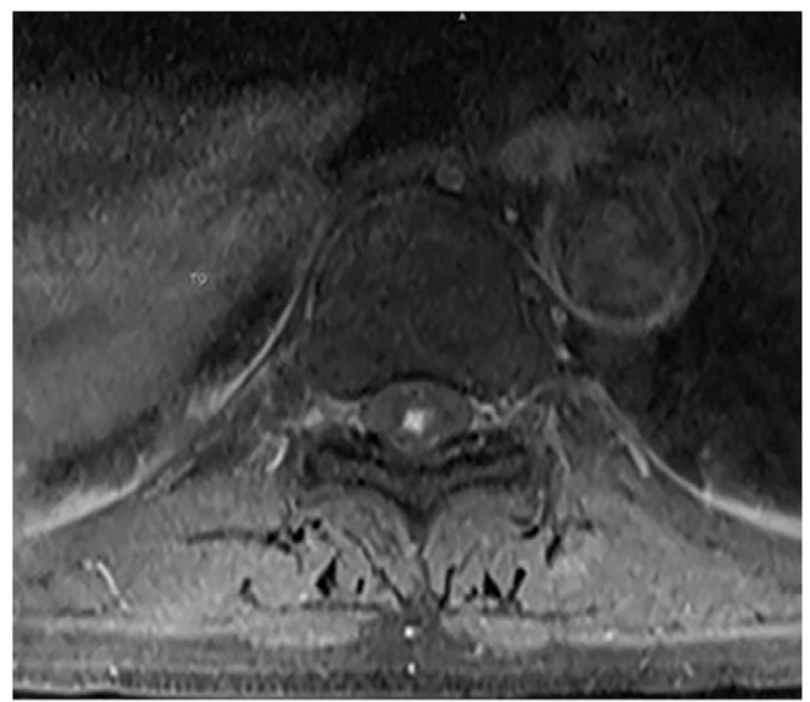

FIGURE 2. Hyperintensity lesion at T10 level in the axial T1 view of the T-spine using magnetic resonance imaging (MRI) with gadolinium.

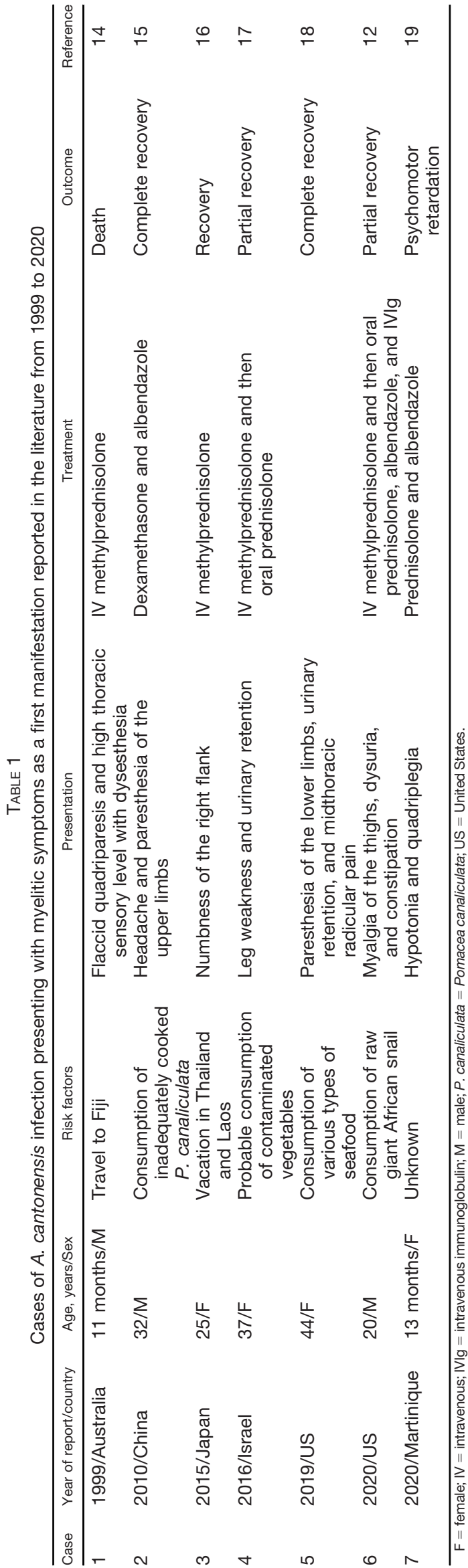


In Thailand, if patients with a history of raw food consumption present with self-limiting radicular pain followed by any myelitic signs at different anatomical levels, then gnathostomiasis is the provisional diagnosis. ${ }^{9}$ This case presentation reflects the migration of larva through the nerve root, thecal sac, and spinal cord. ${ }^{10}$ Although myelitis in neuro-gnathostomiasis is welldocumented, $A$. cantonensis myelitis is rare. A previous case series of 12 patients with $A$. cantonensis infection reported no myelitis. Neither CBC results nor the presence of CSF eosinophilia can be used to distinguish between neurognathostomiasis and neuro-angiostrongyliasis. A spinal MRI will show multiple linear, track-like, scattering punctate lesions with cord swelling with both diseases, but hemorrhagic lesions are more typically found with neuro-gnathostomiasis than with neuro-angiostrongyliasis. ${ }^{11,12}$ Molecular and serologic studies are important to confirm the diagnosis and distinguish between these diseases.

For this case, neither the CBC nor CSF examination revealed eosinophilia; however, $\mathrm{MRI}$ revealed minimal swelling of the spinal cord because its use was delayed. The patient had been admitted for supportive treatment and waited 3 weeks before MRI was performed and a diagnosis was made. The false-negative results of the CSF PCR assay for A. cantonensis may have been attributable to its timing. A diagnosis of neuro-angiostrongyliasis was confirmed by a positive antibody to $A$. cantonensis from an immunochromatographic test (ICT) kit developed by the Department of Parasitology of Khon Kaen University. The accuracy of this test is comparable to that of the standard method, and it is used as the referral method for measuring the accuracy of other test kits. $^{6,13}$ The results of serologic testing for $G$. spinigerum were negative, thereby ruling out neuro-gnathostomiasis. A partial response to dexamethasone and albendazole was observed as expected. The minimal swelling of the spinal cord and absence of CSF pleocytosis indicated that the inflammation caused by $A$. cantonensis had nearly resolved on its own before treatment was initiated.

Received April 29, 2021. Accepted for publication May 10, 2021.

Published online July 16, 2021.

Acknowledgment: We thank Dr. Dylan Southard for editing the manuscript via the KKU Publication Clinic (Thailand).

Disclosure: The patient provided informed consent prior to this report.

Authors' addresses: Tharatorn Nuntawit, Wantin Sribenjalux, and Atibordee Meesing, Division of Infectious Diseases and Tropical Medicine, Department of Medicine, Faculty of Medicine, Khon Kaen University, Khon Kaen, Thailand, E-mails: tnuntawit@hotmail.com, wantinmd34@gmail.com, and atibordee@hotmail.com.

\section{REFERENCES}

1. Barratt J, Chan D, Sandaradura I, Malik R, Spielman D, Lee R, Marriott D, Harkness J, Ellis J, Stark D, 2016. Angiostrongylus cantonensis: a review of its distribution, molecular biology and clinical significance as a human pathogen. Parasitology 143: 1087-1118.
2. Lv S, Zhou X-N, Andrews JR, 2017. Eosinophilic meningitis caused by Angiostrongylus cantonensis. ACS Chem Neurosci 8: 1815-1816.

3. Nash TE, 2020. Visceral larva migrans and other uncommon helminth infections. Bennett JE, Dolin R, Blaser MJ, eds. Mandell, Douglas, and Bennett's Principles and Practice of Infectious Disease, ninth edition. Canada: Elsevier Saunders, 3473-3478.

4. Eamsobhana P, 2013. Angiostrongyliasis in Thailand: epidemiology and laboratory investigations. Hawaii J Med Public Health 72: S28-S32.

5. Khamsai S, Chindaprasirt J, Chotmongkol V, Tiamkao S, Limpawattana P, Senthong V, Sawanyawisuth K, 2020. Clinical features and course of Angiostrongylus cantonensis eosinophilic meningitis in patients receiving supportive therapy. Food Waterborne Parasitol 21: e00095.

6. Somboonpatarakun C, Intapan PM, Sadaow L, Rodpai R, Sanpool O, Maleewong W, 2020. Development of an immunochromatographic device to detect antibodies for rapid diagnosis of human angiostrongyliasis. Parasitol 147: 194-198.

7. Eamsobhana $P, 2010$. Towards precise and rapid diagnosis of eosinophilic meningitis due to the rat lungworm Angiostrongylus cantonensis. Siriraj Med J 62: 84-89.

8. Sawanyawisuth K, Kitthaweesin K, Limpawattana P, Intapan PM, Tiamkao S, Jitpimolmard S, Chotmongkol V, 2007. Intraocular angiostrongyliasis: clinical findings, treatments and outcomes. Trans R Soc Trop Med Hyg 101: 497-501.

9. Katchanov J, Sawanyawisuth K, Chotmongkol V, Nawa Y, 2011. Neurognathostomiasis, a neglected parasitosis of the central nervous system. Emerg Infect Dis 17: 1174-1180.

10. Schmutzhard E, Boongird P, Vejjajiva A, 1988. Eosinophilic meningitis and radiculomyelitis in Thailand, caused by CNS invasion of Gnathostoma spinigerum and Angiostrongylus cantonensis. J Neurol Neurosurg Psychiatry 51: 80-87.

11. Kanpittaya J, Sawanyawisuth K, Intapan PM, Khotsri P, Chotmongkol V, Maleewong W, 2012. A comparative study of neuroimaging features between human neuro-gnathostomiasis and angiostrongyliasis. Neurol Sci 33: 893-898.

12. Widder JR, Fallah S, Mondzelewski TJ, 2010. A case report of slug ingestion causing eosinophilic meningitis, papilledema, and pronounced motor weakness in a US marine. Mil Med 185: 317-321.

13. Graeff-Teixeira C, Pascoal VF, Rodriguez R, Morassutti AL, Intapan PM, Maleewong W, 2020. Abdominal angiostrongyliasis can be diagnosed with a immunochromatographic rapid test with recombinant galactin from Angiostrongylus cantonensis. Mem Inst Oswaldo Cruz 115: e200201.

14. Cooke-Yarborough CM, Kornberg AJ, Hogg GG, Spratt DM, Forsyth JR, 1999. A fatal case of angiostrongyliasis in an 11month-old infant. Med J Aust 170: 541-543.

15. Diao Z, Yin C, Jin E, 2010. Myelitis caused by infection of Angiostrongylus cantonensis. Am J Trop Med Hyg 83: 1176-1177.

16. Ueda M, Takeuchi Y, Ochiai J, Mabuchi C, Niwa J, 2015. A case of myelitis with eosinophilia of the cerebrospinal fluid. Clinica Neurol 55: 651-653.

17. Fellner A, Hellmann MA, Kolianov V, Bishara J, 2016. A non-travel related case of Angiostrongylus cantonensis eosinophilic meningomyelitis acquired in Israel. J Neurol Sci 370: 241-243.

18. Long D, Green K, Derani T, Decker N, Pace RJ, Aburashed R, 2019. CNS-disseminated Angiostrongylus cantonensis mimicking atypical demyelinating disease. Neurol Neuroimmunol $\mathrm{Neu}$ roinflamm 6: e608.

19. Dard C, Tessier E, Nguyen D, Epelboin L, Harrois D, Swale C, Cabié A, De Meuron K, Miossec C, Desbois-Nogardet N, 2020. First cases of Angiostrongylus cantonensis infection reported in Martinique, 2002-2017. Parasite 27: 31. 